\title{
Die Regeln der Eisenhüttenkunde
}

\section{Genese und Struktur eines technikwissenschaftlichen Feldes, 1870-1914}

\author{
Stefan Krebs
}

\begin{abstract}
The Rules of Ferrous Metallurgy. Genesis and Structure of a Field of Engineering Science, 1870-1914
\end{abstract}

\begin{abstract}
The ways in which the sciences have been delineated and categorized throughout history provide insights into the formation, stabilization, and establishment of scientific systems of knowledge. The Dresdener school's approach for explaining and categorizing the genesis of the engineering disciplines is still valid, but needs to be complemented by further-reaching methodological and theoretical reflections. Pierre Bourdieu's theory of social practice is applied to the question of how individual agents succeed in influencing decisively a discipline's changing object orientation, institutionalisation and self-reproduction. Through the accumulation of social, cultural and economic capital, they succeed in realising their own organisational ideas and scientific programs. Key concepts for the analysis include the struggle for power and resources, monopolies of interpretation, and the degree of autonomy. A case study from the Aachener Technische Hochschule shows that the consolidation of ferrous metallurgy can be conceived as a symbolical struggle between Fritz Wüst, professor for ferrous metallurgy, and the German Iron and Steel Institute, leading to a construction of a system of differences in which scientists accepted being scientists rather than entrepreneurs, and entrepreneurs accepted becoming entrepreneurs and renounced science.
\end{abstract}

Keywords: ferrous metallurgy, genesis of disciplines, engineering sciences, social practice, Pierre Bourdieu, Fritz Wüst, German Iron and Steel Institute

Schlüsse/wörter: Eisenhüttenkunde, Disziplingenese, Technikwissenschaften, soziale Praxis, Pierre Bourdieu, Fritz Wüst, Verein Deutscher Eisenhüttenleute 


\section{Vorbemerkungen}

Zwei Beobachtungen dienen als Ausgangspunkt der folgenden Überlegungen. Zum einen nahm die Aachener Eisenhüttenkunde von der Gründung des Aachener Polytechnikums 1870 bis um die Jahrhundertwende trotz der Nähe zum rheinisch-westfälischen Industriegebiet keine herausragende Position innerhalb der deutschen Hüttenkunde ein, avancierte dann aber binnen eines einzigen Jahrzehnts zum größten und angesehensten Studien- und Forschungsstandort. Hatte 1901 der neu berufene Professor für Eisenhüttenkunde, Fritz Wüst ${ }^{1}$, noch den schlechten Zustand seines Fachs in Aachen beklagt, verkündete der Aachener Abgeordnete Franz Kaufmann schon im April 1910 voller Stolz im Preußischen Abgeordnetenhaus: Das Aachener Institut für Eisenhüttenkunde sei „die erste Anstalt von Europa“2. Zwischen 1901 und 1910 müsste demnach eine besonders dynamische Entwicklung der Eisenhüttenkunde in Aachen stattgefunden haben.

Zum anderen notierte Fritz Wüst 1925 rückblickend über die Veränderungen der Eisenhüttenkunde zwischen der Jahrhundertwende und dem Ersten Weltkrieg: „Die Erfahrung musste ihre Jahrhunderte alte Herrschaft mit einer neuen Macht, der Wissenschaft, teilen, deren richtige Anwendung für das wirtschaftliche Gedeihen der Hüttenwerke von stets zunehmender Bedeutung wurde." ${ }^{\text {33 }}$ Aus diesem Resümee spricht zunächst das Selbstbewusstsein des erfolgreichen Wissenschaftlers, dann lässt sich aber auch herauslesen, dass sich der Aufstieg der wissenschaftlichen Eisenhüttenkunde nur im Konflikt mit der eisenhüttenmännischen Praxis vollziehen konnte.

Aus diesen kurz skizzierten Entwicklungslinien ergeben sich zwei miteinander verzahnte Leitfragen:

- Erstens ist nach den Gründen für den außerordentlichen Erfolg der Aachener Eisenhüttenkunde zu fragen; dabei geht es darum, die kognitive und soziale Disziplinentwicklung der Eisenhüttenkunde zu (re-)konstruieren.

- Zweitens ist der Konflikt zwischen der eisenhüttenkundlichen Wissenschaft und der industriellen Praxis zu untersuchen.

Übergreifend werden dabei die Besonderheiten der technikwissenschaftlichen Disziplingenese und die Veränderungen der sozialen Praxis der Eisenhüttenkunde herausgearbeitet. Zur Bearbeitung dieses Fragenkomplexes werden zwei methodisch- 
theoretische Herangehensweisen miteinander verschränkt, die sich gegenseitig ergänzend - die Entwicklung der Eisenhüttenkunde sowie den Aufstieg des Aachener Instituts erklären helfen: das Dresdener Konzept zur Genese technikwissenschaftlicher Disziplinen (Guntau/Laitko 1987a, Hänseroth/Mauersberger 1996) und die Theorie der Praxis des französischen Soziologen Pierre Bourdieu (1992, 1998, 2001a).

Im Mittelpunkt des Dresdener Konzepts stehen drei Untersuchungsebenen: die Gegenstandsorientierung einer Disziplin, ihre Institutionalisierung sowie ihre Selbstreproduktion. Unter Gegenstandsorientierung, das heißt unter der Orientierung wissenschaftlicher Tätigkeit auf einen bestimmten Erkenntnisgegenstand, wird das systembildende Prinzip wissenschaftlicher Disziplinen verstanden. Der Wandel der kognitiven Erkenntnisziele und -dispositionen allein erklärt aber noch nicht das Zustandekommen und vor allem die Permanenz einzelner Disziplinen, vielmehr hat die Neubildung einer Disziplin notwendige institutionelle Voraussetzungen und Konsequenzen. Neben anderen Institutionstypen werden insbesondere produzierende und reproduzierende Institutionen unterschieden: Erstere sind auf die Produktion wissenschaftlicher Erkenntnisse ausgerichtet, letztere dienen der lehrhaften Vermittlung dieser Erkenntnisse an den wissenschaftlichen Nachwuchs. Eine zentrale Funktion des Institutionalisierungsprozesses liegt darin, ,,die Permanenz des disziplinären Tätigkeitssystems durch Reproduktion seines Potentials zu gewährleisten“ (Guntau/Laitko 1987b: 39).

Zur Methodik des Dresdener Konzepts gehört wesentlich die Erarbeitung eines Periodisierungsschemas, das voneinander $\mathrm{zu}$ unterscheidende Stadien der Genese von Technikwissenschaften beschreibt. So wird vor allem zwischen einer Herausbildungs- und einer Konsolidierungsphase unterschieden (Abb. 1). Die Phase der Herausbildung ist geprägt von sammelnder Detailforschung, der Systematisierung des Erfahrungswissens und dessen lehrhafter Vermittlung, während die Phase der Konsolidierung von experimenteller Laborforschung und der kognitiven Verwissenschaftlichung der Disziplin, verstanden als Dominanz antizipativer Theoriebildung, gekennzeichnet ist (Hänseroth/Mauersberger 1996, Buchheim 1984, König 1995: 6).

Diese Überlegungen zur Genese technikwissenschaftlicher Disziplinen sollen anhand der Aachener Hochschule auf das Fallbeispiel der Eisenhüttenkunde übertragen werden. Dabei wird gezeigt, welche Stellung die Aachener Eisenhüttenkunde zwischen 


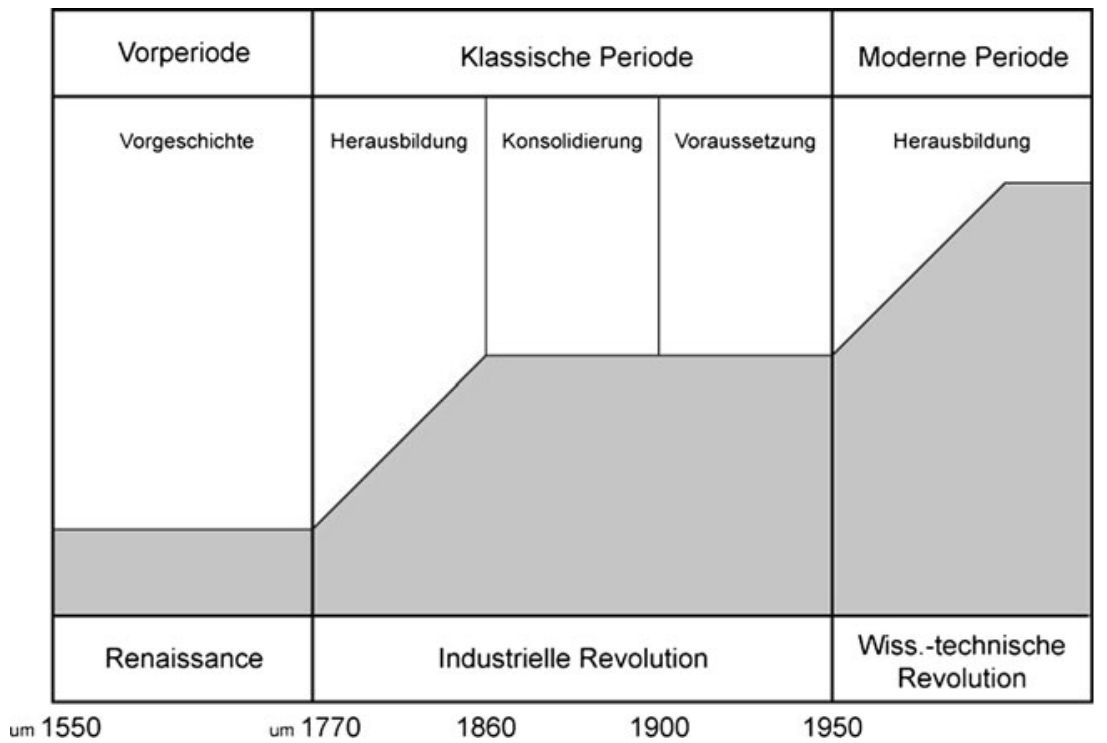

Abb. 1: Dresdener Periodisierungsschema zur Genese der Technikwissenschaften (Hänseroth/Mauersberger 1996: 40).
1870 und dem Ausbruch des Ersten Weltkriegs innerhalb der gesamten Disziplinentwicklung in Deutschland einnahm. Das heißt, das Aachener Fallbeispiel wird nicht als unabhängige lokale Disziplingenese begriffen, sondern in den Kontext der Entwicklung an den anderen deutschen Studienstandorten eingebettet. Die gewählte Mikroperspektive erlaubt es aber, die Geschehnisse auf der Ebene einzelner Personen und Dokumente, das soziale Handeln im Feld der Eisenhüttenkunde nachzuvollziehen.

Als eine Art blinder Fleck des Dresdener Konzepts bleibt die Frage offen, wie es den handelnden Akteuren gelingt, maßgeblichen Einfluss auf den Verlauf und die Richtung der Entwicklung einer Disziplin zu gewinnen. Zur Ergänzung bietet sich Bourdieus Theorie der sozialen Praxis an. Für ihn ist das universitäre Feld eine Stätte des Kampfes um Klassifizierungen und Rangordnungen - den Regeln der Einzelwissenschaften beziehungsweise des übergeordneten wissenschaftlichen Feldes. Die jeweils in einem historischen Moment bestehenden Kräfteverhältnisse bestimmen darüber, ob sich einzelne Akteure mit ihren Deutungen durchsetzen, hierdurch das Feld verändern beziehungsweise für den Erhalt der herrschenden Ordnung sorgen können (Bourdieu 1992: 55). Ähnliche Überlegungen finden sich auch bei Mitchell G. Ash und Ulrike Felt, die vorschlagen, Wissenschaften als Ressourcenensemble aufzufassen. Die Verfügung über materielle und immaterielle Ressourcen sind dann hinreichende Bedingungen dafür, dass einzelne Akteure gestaltend Einfluss auf die 
Wissenschaftsentwicklung nehmen können (Ash 2002, Felt 2002). Zur Operationalisierung dieses Konzepts stellt Bourdieus Kapitaltheorie ein elaboriertes Set an Begriffen für die Analyse der Feldstruktur der Eisenhüttenkunde bereit: Insbesondere die von ihm unterschiedenen Kapitalsorten sind hilfreich, um die materiellen und immateriellen Ressourcen zu beschreiben, mit deren Hilfe die einzelnen Akteure ihre Positionen und damit ihre Machtstellungen verbessern können oder durch deren Verlust sie an Einfluss verlieren (Bourdieu 1983). Bourdieu selbst hat durch seine Untersuchung des literarischen Feldes in Frankreich zu Beginn des 19. Jahrhunderts gezeigt, wie seine Kapitaltheorie für eine historische Untersuchung herangezogen werden kann (Bourdieu 2001a). Zudem hat er in seinem Homo academicus (1992) die besonderen Regeln der Wissenschaft luzide und überzeugend beschrieben. Bourdieu versteht diese als die grundlegenden Klassifizierungen und Ordnungen eines Feldes, die darüber entscheiden, ob bestimmte Handlungen und Erkenntnisse als legitim und relevant anerkannt werden. Dagegen geht es ihm nicht darum, welche Grundsätze im Sinne konkreter Handlungsanweisungen der Durchführung einzelner wissenschaftlicher Untersuchungen zugrunde liegen können.

Wissenschaft ist für Bourdieu ein Feld eingeschränkter Produktion: ein Raum der Wissensproduktion, ,in dem das Publikum der Produzenten im wesentlichen aus anderen Produzenten, also unmittelbaren Konkurrenten besteht" (Champagne 1998: 11). Zunächst ist Wissenschaft eine soziale Welt wie andere auch, sie folgt aber mehr oder weniger spezifischen Regeln:

Obwohl [der Raum der Wissenschaft] sich nie ganz den Zwängen des Makrokosmos entziehen kann, verfügt er doch über eine mehr oder weniger ausgeprägte Autonomie. Eine der großen Fragen, die sich im Bezug auf wissenschaftliche Felder [...] stellen wird, betrifft eben den Grad der Autonomie, über die sie verfügen können. (Bourdieu 1998: 18)

Die relative Unabhängigkeit von wirtschaftlichen und politischen Machtinstanzen ist demnach für Bourdieu ein wesentlicher Teil des Nomos des wissenschaftlichen Feldes.

Wissenschaftswandel ist also ein Kampf um Deutungen und Deutungssysteme. Die Akteure, die miteinander um die Veränderung oder den Erhalt der aktuellen Struktur eines wissenschaftlichen Feldes kämpfen, können aufgrund ihrer Kapitalausstattung, ihrer jeweiligen Position innerhalb des Feldes sowie ihrer habituellen Dispositionen Strategien zur Durchsetzung ihrer Positionierungen verfolgen. Sie zielen darauf, selbst beherrschende Positionen innerhalb des Feldes zu erlangen beziehungsweise zu 
behalten, um so möglichst großen Einfluss auf die wissenschaftliche Entwicklung zu gewinnen. Schlüsselbegriffe für die Analyse wissenschaftlicher Felder sind demzufolge der Kampf um Macht, Ressourcen und Deutungsmonopole sowie um den Grad an Autonomie einzelner Disziplinen.

Aus der Verknüpfung des Dresdener Konzepts mit Bourdieus Theorie der Praxis ergeben sich vier Leitgedanken:

- Disziplingenese ist erstens wissenschaftlicher Wandel, der im Fall der Technikwissenschaften in Phasen abläuft, deren Übergänge sich zwar durchaus fließend gestalten können, die aber nichtsdestoweniger klar voneinander zu unterscheiden sind.

- Disziplingenese ist zweitens der Wandel von Ressourcenkonstellationen, der durch die Positionen und Dispositionen einzelner Akteure beeinflusst wird.

- Drittens ist die Disziplingenese eine soziale Praxis, die sich innerhalb des akademischen Feldes und in Kooperation und Abgrenzung $\mathrm{zu}$ benachbarten und überlagernden Feldern herausbildet.

- Viertens ist Disziplingenese ein Autonomisierungsprozess einzelner disziplinärer Felder oder dem darüber liegenden Feld der Wissenschaft. Insgesamt ermöglicht die Untersuchung der Feldstruktur, die historisch kontingente Wahrheit verschiedener Positionen zu verstehen und die Grenzen der Gültigkeit unterschiedlicher Stellungnahmen aufzuzeigen.

Bourdieus Theorie der Praxis kann also helfen, die im Rahmen des Dresdener Ansatzes beschriebenen Phasen der technikwissenschaftlichen Disziplingenese als historisch erklärbaren Strukturwandel eines Feldes zu begreifen, der durch die sich wandelnden Kräfteverhältnisse, die Positionen und Positionierungen der auf ihm agierenden Akteure sowie die von diesen durchgesetzten oder bekämpften Regeln verläuft. Die im Dresdener Modell implizit enthaltene These einer teleologischen Entwicklung der Technikwissenschaften zu konsolidierten, auf eigener sozialer und kognitiver Basis stehenden Disziplinen wird damit in Frage gestellt. Vielmehr zeigt die mikrohistorische Untersuchung der Machtverhältnisse auf dem Feld der Eisenhüttenkunde, dass die langanhaltende Orthodoxie der ausschließlichen Orientierung auf die industrielle Praxis, die häretische Herausforderung dieses Denkstils und die damit einsetzende Autonomisierung wesentlich von der Kapitalausstattung und den Strategien der zentralen Akteure abhängig waren. Der Werkzeugkasten Bourdieus wird insofern nicht als bloße Ergänzung des 
Dresdener Ansatzes, sondern zugleich als kritisches Korrektiv verstanden.

\section{Herausbildungsphase (1871-1900)}

Während die Vorgeschichte der eisenhüttenkundlichen Disziplingenese bis ins 16. Jahrhundert zurückreicht, setzte die Herausbildungsphase mit der Gründung der Bergakademien beziehungsweise deren Vorgängerinstitutionen in den 1760er Jahren ein (Krebs 2009: 51-60). Die in Aachen praktizierte Vertretung der Metall- und Eisenhüttenkunde als ein einziges großes Gegenstandsgebiet war im Grunde schon bei der Errichtung des Lehrstuhls 1871 anachronistisch. An den drei deutschen Bergakademien wurden zwischen 1863 und 1885 eigene Lehrstühle für Eisenhüttenkunde eingerichtet, während die Technischen Hochschulen nur je einen Vertreter für die gesamte Hüttenkunde hatten. An der Aachener Hochschule gab es bis 1897 für die Hüttenkunde nur einen einzigen Lehrstuhl (ebd.: 52-54, Hoffmann 1959: 73, 81, Schwarz 2000: 580-589).

Da die Bergakademien eine unliebsame Konkurrenz verhindern wollten, hatte das preußische Handelsministerium zunächst vollkommen auf die Einrichtung eines hüttenkundlichen Lehrstuhls an der 1870 eröffneten Aachener Hochschule verzichtet. Erst die Initiative der rheinisch-westfälischen Stahlindustrie, die auch eine Anschubfinanzierung in Aussicht stellte, überzeugte das Ministerium, zum 1. November 1871 eine entsprechende Dozentur einzurichten (Düwell 1970: 57, Wüst 1905). Trotz dieser ungünstigen Ausgangssituation durch die um ein Jahr verzögerte Errichtung des Lehrstuhls entwickelte sich die Disziplin zunächst gut: Der erste Lehrstuhlinhaber, Ernst Friedrich Dürre ${ }^{4}$, konnte rasch eigene reproduktive Institutionen in Form einer metallurgischen Sammlung und vor allem eines Probierlaboratoriums aufbauen (TH Aachen 1879, Dürre 1877). Im Vergleich zu den anderen Disziplinen an der Aachener Hochschule rückte die Hüttenkunde, abgesehen von der personellen Ausstattung, hinter den chemischen Fächern rasch an die zweite Stelle auf. Die anderen Fächer außer Chemie und Hüttenkunde verfügten in den ersten Jahren über gar keine eigenen Laboratorien, vielmehr teilten sie sich die Vorlesungs- und Zeichensäle im Hauptgebäude. Im Unterschied zur Chemie verfügte die Eisenhüttenkunde jedoch nur über ein Probierlaboratorium, das ganz auf die lehrhafte 
Vermittlung der in der Praxis üblichen chemischen Analyseverfahren ausgerichtet war. ${ }^{5}$

Dürres Lehrveranstaltungen, die Ausstattung des Probierlaboratoriums sowie auch seine Forschungsarbeiten - zusammengefasst in seinen Lehr- und Handbüchern - befanden sich, soweit sich dies feststellen lässt, auch international auf der Höhe ihrer Zeit (Dürre 1870, 1882-1892, Anonym 1887, Anonym 1892, Ledebur 1893, Krebs 2009: 73-90). Aufgrund mehrerer Studienreisen durch die deutschen, belgischen, französischen und englischen Hüttenreviere hielt Dürre den Kontakt zum aktuellen Stand der Hüttentechnik. Zudem besuchte er zahlreiche in- und ausländische Unterrichtsanstalten für Hüttenleute, informierte sich über die neuesten Unterrichtsgegebenheiten vor Ort und adaptierte diese in seine eigene Lehre. ${ }^{6}$ Die geringen Anteile der Hüttenkunde am Curriculum des Studiengangs und die unklaren Grenzen zur analytischen Chemie zeigen aber, dass die Hüttenkunde trotz des Ausbaus ihres eigenen institutionellen Ressourcenensembles durchaus noch als Teil der Technischen Chemie und damit nicht als eine auf selbständiger Grundlage stehende Disziplin angesehen wurde.

Dürre war anfänglich gut in die Scientific Community der Eisenhüttenkunde integriert. Er engagierte sich nachdrücklich in den lokalen und überregionalen technischen Fachvereinen. So wurde ihm innerhalb des Technischen Vereins für das Eisenhüttenwesen das Amt der Schriftleitung übertragen, wodurch er eine wichtige institutionelle Schaltstelle für sich einnahm. Durch die Mitherausgabe der Zeitschrift des Vereins Deutscher Ingenieure, einer weiteren Autoritätsstellung, gehörte Dürre zu den mächtigsten Vertretern der wissenschaftlichen Eisenhüttenkunde. Auf dem im Grunde hybriden Feld der Eisenhüttenkunde, in dem noch keine feste Grenze zwischen Wissenschaft und Wirtschaft gezogen wurde (König 2006: 38), gehörte er aber zu den ,beherrschten Herrschenden“ (Bourdieu 1987: 287). Die Dominanz der wirtschaftlichen Seite des Feldes ist unter anderem an den Vorsitzenden des Technischen Vereins für das Eisenhüttenwesen ablesbar, die alle dem ökonomischen Feld angehörten. ${ }^{7}$

Im Jahr 1878 beschädigte ein Streit mit Joseph Schlink, Direktor der Friedrich-Wilhelms Hütte und einflussreiches Mitglied des Technischen Vereins für das Eisenhüttenwesen, Dürres Position und Ansehen nachhaltig. Schlink erklärte in einem Vortrag vor den Mitgliedern des Technischen Vereins die Theoriebildung der Eisenhüttenkunde für überflüssig und nutzlos. 
Er kritisierte namentlich Dürres Lehrveranstaltungen als theoriebeladen und damit wirklichkeitsfremd (Schlink 1878a). Auf die Veröffentlichung dieser für ihn infamen Vorwürfe in den Annalen für Gewerbe und Bauwesen reagierte Dürre seinerseits mit einer polemischen Zuschrift, in der er Schlinks Kritik heftig zurückwies (Dürre 1878). Dieser antwortete wiederum umgehend darauf und machte sich über Dürre und die für ihn abgehobene weltfremde Unterrichtspraxis der Aachener Hochschule lustig (Schlink 1878b). Dürre verzichtete schließlich auf eine erneute Replik, zumal ihn auch keiner seiner Fachkollegen aus Berlin, Freiberg oder Clausthal unterstützte - im Gegenteil: Sein akademischer Lehrer, Hermann Wedding, distanzierte sich von seinem Schüler und beeilte sich zu versichern, dass der eisenhüttenkundliche Unterricht in Berlin ganz praxisnah gestaltet sei (Wedding 1878). Der ausbleibende Beistand seitens der anderen Hochschullehrer dürfte zum Teil auf deren Furcht vor einem Ordnungsruf der mächtigen Industrievertreter beruht haben. Daneben ist er Ausdruck des traditionellen Wissenschaftsverständnisses an den Bergakademien und der Berliner Technischen Hochschule: Vordringliches Ziel war die lehrhafte Vermittlung des praktischen Erfahrungswissens und nicht die theoretische Durchdringung der eisenhüttenkundlichen Interessensgegenstände - ein typisches Merkmal der Herausbildungsphase.

Im Unterschied zur Theoriedebatte im Maschinenbau, die angestoßen von Ferdinand Redtenbachers Prinzipien der Mechanik (1852) - ihren Höhepunkt mit Franz Reuleauxs Theoretischer Kinematik (1875) erreichte und zur Jahrhundertwende bereits wieder abebbte, setzte in der Eisenhüttenkunde keine Diskussion über ihre Methoden und Ziele innerhalb des wissenschaftlichen Feldes ein. Das Primat der industriellen Praxis war noch so dominierend, dass der Eisenhüttenkundler Wedding Schlink sogar in seiner Kritik der Theorielastigkeit öffentlich sekundierte. Zudem verblieb auch Friedrich Dürre der sammelnden Detailforschung verhaftet, und die von ihm ermittelten Koeffizienten und Verhältniszahlen verließen im Grunde das Prinzip der Systematisierung des Erfahrungswissens nicht. Schlinks scharfe Reaktion ist daher weniger Dürres tatsächlicher Lehr- und Forschungstätigkeit geschuldet, vielmehr suchte er nach einem konfliktreichen Thema, mit dem er die Loslösung des Technischen Vereins für das Eisenhüttenwesen vom Verein Deutscher Ingenieure forcieren konnte. Dass er sich dabei auf Dürre kaprizierte, war nach eigenem Bekunden ganz zufällig, da er bei der 
Abfassung seines Redemanuskripts nur das Aachener Vorlesungsprogramm zur Hand gehabt habe (Krebs 2009: 89, 102-112, 114, Hänseroth/Mauersberger 1998: 229-233).

Dürre reagierte auf den Streit mit seinem Rückzug aus sämtlichen Ämtern und Funktionen, womit er einen Großteil seines sozialen und wissenschaftlichen Kapitals sowie seine institutionellen Positionen außerhalb Aachens verlor. Besonders dramatisch dürfte der Abbruch der Beziehungen zum neugegründeten Verein Deutscher Eisenhüttenleute gewesen sein, da Dürre damit nicht nur einen Teil seines eigenen sozialen Kapitals einbüßte, sondern, im Sinne von Bourdieus Multiplikatoreffekt des sozialen Kapitals, auch das soziale, ökonomische und symbolische Kapital des Fachvereins (Milkereit 1977). Zudem verweigerte er die Mitarbeit an der neuen Vereinszeitschrift Stahl und Eisen. Diese entwickelte sich, besonders durch die intensive literarische Mitarbeit von Hermann Wedding und Adolf Ledebur, zum zentralen Fachorgan für die Eisenhüttenkunde - was wechselseitig das Ansehen der beiden Professoren und der Zeitschrift mehrte. Friedrich Dürre und die Aachener Eisenhüttenkunde rückten damit zunehmend an die Peripherie des Feldes, obschon sich die Hochschule geographisch als einziger Studienstandort in direkter Nähe zum rheinisch-westfälischen Industriegebiet befand.

Während Dürres Reputation und Einfluss außerhalb Aachens schwanden, wurde seine Stellung innerhalb des Kollegiums offensichtlich nicht erschüttert. Vielmehr waren sein Ansehen und seine Beziehungen zu den Kollegen so gut, dass er mehrfach wichtige Funktionen übertragen bekam und sogar für drei Jahre zum Rektor gewählt wurde. Jedoch gelang es ihm nicht, diese Machtstellung für den Ausbau seiner Disziplin einzusetzen. Zum Nachteil der von ihm vertretenen Eisenhüttenkunde fühlte er sich der dem wissenschaftlichen Feld konstitutiven Uneigennützigkeit bei der Ausübung seiner Ämter verpflichtet und verkannte insofern die tatsächlichen Spielregeln des akademischen Feldes. Seine Aachener Kollegen hatten weniger Scheu, ihre akademischen Machtpositionen rücksichtslos zu ihren eigenen Gunsten auszunutzen: Alexander Classen, Professor für anorganische Chemie, erstritt vehement einen größeren Anteil an der Ausbildung der Chemiker und Hüttenleute für sich und damit einen wesentlich höheren Anteil an den Unterrichtshonoraren. Die Professoren für Bergbaukunde, Wilhelm Schulz, und Geologie und Paläontologie, Eduard Holzapfel, nutzten ihre Stellung als Senatoren aus, um den von ihrer Abteilung auf Platz eins gesetzten Antrag auf Schaffung 
einer Dozentur für Metallhüttenkunde zugunsten eines eigenen Stellengesuchs auf Platz zwei zu verdrängen (Krebs 2009: 123130). Dürres fehlender Praxissinn war insofern mit ein Grund für die zwei Jahrzehnte andauernde Stagnation der hüttenkundlichen Disziplingenese in Aachen.

Die anhaltende Praxisorientierung des Eisenhüttenwesens hemmte zudem die weitere Verwissenschaftlichung und damit den Übergang zur Konsolidierungsphase. Dürres Amtsnachfolger Fritz Wüst beklagte noch 1907 das Theoriedefizit der deutschen Eisenhüttenkunde angesichts der Fortschritte, die in den vergangenen zwanzig Jahren in Belgien, Frankreich, England und den Vereinigten Staaten erzielt worden seien. ${ }^{8}$

\section{Konsolidierungsphase (1901-1914)}

Nachdem die Aachener Eisenhüttenkunde aufgrund von Dürres Positionsverlust seit den 1880er Jahren eine eher randständige Position auf dem eisenhüttenkundlichen Feld in Deutschland eingenommen hatte, übernahm sie nach der Jahrhundertwende die Rolle der eisenhüttenkundlichen Avantgarde. Und noch vor dem Ersten Weltkrieg setzte sie ihre spezifische Ausprägung als die arrivierte Position durch, an der sich die anderen eisenhüttenkundlichen Akteure orientieren mussten.

In dieser stürmischen Aufbauzeit setzte Fritz Wüst, der 1901 die Nachfolge von Friedrich Dürre antrat, grundlegende Änderungen der von ihm vertretenen Disziplin durch: eine veränderte Gegenstandsorientierung, neue Forschungsmethoden und -ziele, den Auf- und Ausbau von Institutionen, erste Schritte zur Selbstreproduktion und schließlich die erfolgreiche Verstetigung der Wüst'schen Schule. Insgesamt verzeichnete die Aachener Eisenhüttenkunde zwischen 1901 und 1914 eine gewaltige Expansion ihres gesamten Ressourcenensembles - nicht zuletzt zu Lasten anderer technikwissenschaftlicher Disziplinen und Studienstandorte. In Aachen verlor beispielsweise die anorganische Chemie große Unterrichtsanteile an der Ausbildung von Hütteningenieuren, womit Classen einen Teil seiner zuvor erkämpften Unterrichtshonorare einbüßte. Zum Studienjahr 1905/1906 brachen an den beiden Berliner Anstalten die Einschreibungen für Hüttenkunde ein, während sie sich in Aachen leicht erhöhten. Prozentual stieg der Aachener Studiengang in diesem Studienjahr von $34 \%$ auf $52 \%$ aller Einschreibungen für Hüttenkunde an. 


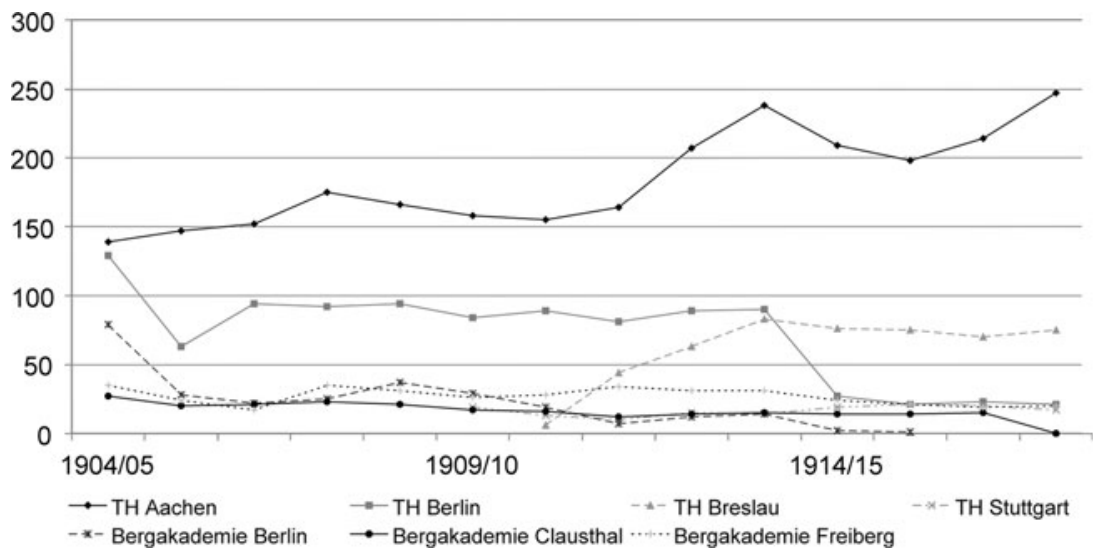

Abb. 2: Zahlen der Hüttenkundestudenten an den Technischen Hochschulen und Bergakademien, 1904-1918 (nach den statistischen Angaben in Stahl und Eisen 1904-1933).
In den folgenden Jahren bis zum Ersten Weltkrieg lagen die Zahlen relativ konstant um die 50\% und stiegen während des Krieges sogar bis auf 59\% an (Abb. 2).

Eigentliche Keimzelle der Konsolidierung der Eisenhüttenkunde war die 1902 vom Verein Deutscher Eisenhüttenleute angestoßene Studienreform. ${ }^{9}$ Sie legte die notwendige Voraussetzung auf sozialer und kognitiver Ebene, um die Eisenhüttenkunde auf ein eigenes institutionelles und methodisch-theoretisches Fundament zu stellen. Die neue Gegenstandsorientierung konzentrierte sich auf drei Bereiche:

- die Maschinentechnik des Eisenhüttenwesens, der mehr Aufmerksamkeit durch einen gesonderten Spezialunterricht geschenkt wurde;

- die physikalische Chemie, die als theoretisches Fundament der Verwissenschaftlichung diente;

- die Stärkung des eisenhüttenkundlichen Fachunterrichts mit dem Schwerpunkt auf praktischen Übungen, bei denen auch neue Methoden - zum Beispiel die Metallographie - eingeübt werden sollten.

Hinzu kam eine deutliche Verringerung der Unterrichtsstunden in der anorganischen Chemie, die in der Diplomprüfung nicht berücksichtigt wurde.

Es lassen sich wenigstens drei Motive für die Neuausrichtung des eisenhüttenkundlichen Curriculums erkennen, die sich im Normalstudienplan während der zweiten Berliner Unterrichtskonferenz im Januar 1904 manifestierten. Zunächst ging die Verbreiterung und Spezialisierung der maschinentechnischen Ausbildung der Eisenhütteningenieure auf einen Wunsch der Industrie zurück, die eine Antwort auf die sich wandelnden 
betrieblichen Anforderungen der Massenstahlherstellung suchte (Wengenroth 1993, Grübler 1996). Ferner war die Zurückdrängung der anorganischen Chemie ein notwendiger emanzipatorischer Schritt, um die Hüttenkunde aus dem Schatten der Chemie herauszuführen und als eigenständige Disziplin zu begründen. Schließlich zielte die gesamte Reorganisation des Studiums auf eine fortgeschrittene Spezialisierung der Ausbildung - mit dem Ergebnis, unter anderem einen eigenen Abschluss anbieten zu können, der die Eisenhütteningenieure sowohl von den Chemikern als auch von den Maschineningenieuren abgrenzte und ihnen entsprechende Karrieremöglichkeiten in der Eisenhüttenindustrie sowie an den Hochschulen und Forschungseinrichtungen eröffnete. Innerhalb der akademischen Selbstverwaltung und der institutionellen Studienorganisation stand das Recht der Hüttenkunde im Vordergrund, weitgehend selbständig über Ausbildungsfragen der Disziplin entscheiden zu können. ${ }^{10}$

Während der beiden Berliner Unterrichtskonferenzen im November 1903 und Januar 1904 erreichte Wüst, dass seine Vorstellungen - auch gegen die Einwände seiner Berliner, Freiberger und Clausthaler Kollegen - als verbindlich festgeschrieben wurden und zunächst nur in Aachen eingeführt werden sollten. Hierbei kann die Unterstützung des Vereins hinsichtlich der Durchsetzung der Interessen der Aachener Eisenhüttenkunde kaum überschätzt werden: Nur die Interessensymbiose zwischen den Aachenern und dem Verein, die Wüst durch intensive Beziehungsarbeit herstellte, ermöglichte es, dass er sich - gerade einmal drei Jahre nach seiner Berufung - gegen seine etablierten Fachkollegen in Aachen und an den anderen Studienstandorten durchsetzen konnte (vgl. Krebs 2009: 207-225, 255-263).

Wüst nutzte die geographische Nähe Aachens zum Vereinssitz in Düsseldorf zu häufigen persönlichen Kontakten. Dies war seinen Berliner, Freiberger und Clausthaler Kollegen verwehrt. Er setzte sich zudem kurz nach seiner Berufung für die Verleihung der Ehrendoktorwürde an den Vereinsvorsitzenden Carl Lueg und den Geschäftsführer Emil Schrödter ein. Mit diesem Schritt kooptierte Wüst die beiden symbolisch ins wissenschaftliche Feld und verpflichtete sie zur Unterstützung. ${ }^{11}$ Ferner nutzte er die Verbindungen $\mathrm{zu}$ einigen von Dürres Schülern, die wichtige Positionen in der rheinisch-westfälischen Stahlindustrie wie dem Verein bekleideten, darunter Friedrich Springorum, seit 1891 Betriebsdirektor des Eisen- und Stahlwerks Hoesch in Dortmund $^{12}$, Fritz Kintzlé, seit 1899 Betriebsdirektor des Aachener 
Hütten-Aktien-Vereins Rothe Erde, und Gisbert Gillhausen, seit 1893 Vorstand des Technischen Büros der Firma Krupp in Essen. Wichtigstes Pfund während der Verhandlungen in Berlin war die Summe von 100.000 Mark, die der Verein Deutscher Eisenhüttenleute auf Betreiben Wüsts für den Neubau eines Instituts an der Technischen Hochschule Aachen in Aussicht stellte (Vorstand 1903). Das Geld stammte aus den Überschüssen der Düsseldorfer Industrie- und Gewerbeausstellung von 1902 und wurde im Verlauf der Verhandlungen durch Spenden der rheinisch-westfälischen Stahlindustrie auf 470.000 Mark erhöht. ${ }^{13}$

Im Rahmen der Berliner Konferenzen verwiesen die beteiligten Industriellen immer wieder auf die rasante Entwicklung der deutschen Eisen- und Stahlindustrie (so habe man 1894 Großbritannien erstmals bei der Stahlerzeugung übertroffen) sowie auf den andauernden scharfen internationalen Wettbewerb. Der Ausbau des höheren hüttenmännischen Unterrichtswesens sei daher eine Überlebensfrage, da die natürliche Benachteiligung Deutschlands mit Rohstoffen nur durch die Arbeit der Ingenieure wettgemacht werden könne - derzeit laufe die Wissenschaft der industriellen Praxis jedoch hinterher. Eine Förderung der Eisenhüttenkunde diene also letztlich dem Gemeinwohl und müsse von daher im Interesse des Staates liegen. ${ }^{14}$ Dieses Resonanzkalkül verfing aber nicht ohne Weiteres, besonders der Vertreter des Finanzministeriums verwies auf die Eigeninteressen der Stahlindustrie und bestand auf einem höheren Eigenanteil, der dann durch Beiträge der Hüttenwerke auch geleistet wurde.

Ferner zeigt beispielsweise die scharfe Auseinandersetzung zwischen Wüst und dem Aachener Industriellen Adolf Kirdorf 1909, dass die eisenhüttenmännische Praxis entgegen ihrer Bekenntnisse während der Verhandlungen die Technikwissenschaften nicht ohne Weiteres als eigenständige Produktivkraft anerkannte. Im Zweifelsfall reklamierte die Praxis das Vorrecht, darüber zu entscheiden, was wissenschaftlich wahr sei. Im Streit um die Zukunft des Thomasverfahrens insistierte Kirdorf beispielsweise, dass wahr nur sei, was sich im Betrieb bewährt habe und damit ökonomisch nützlich sei. Der von Kirdorf initiierte Boykottbeschluss des gesamten Stahlwerksverbands gegen die Aachener Hochschule zeigt, dass diese Meinung auch von anderen Vertretern der Praxis durchaus geteilt wurde (vgl. Krebs 2007: 222-225).

Aufgrund der Vorreiterrolle der Aachener Hochschule sicherte der Abschluss des neuen Studiengangs den Absolventen einen Ausbildungsvorsprung und damit gute Karrierechancen. Der 
Zustrom von Studierenden nach Aachen, der zum Studienjahr 1916/17 seinen Zenit erreichte (vgl. Abb. 2), verweist zum einen auf den Reputationsgewinn der Wüst'schen Schule aufgrund der Durchsetzung der Studienreform und macht zum anderen deutlich, dass sich die Aachener Absolventen beste Voraussetzungen für eine erfolgreiche Karriere im Eisenhüttenwesen ausrechneten.

Personelle und gegenstandsorientierte Ausdifferenzierung der Eisenhüttenkunde bedingten einander - zumal die subjektive „Beherrschung der Komplexität“ (Guntau/Laitko 1987b: 47) nur noch für ein Spezialgebiet innerhalb der Disziplin gegeben war. Die drei großen Bereiche - der chemisch-metallurgische, physikalischmetallographische und konstruktive Teil der Eisenhüttenkunde waren nicht länger durch einen Fachvertreter in Lehre und Forschung beherrschbar; hinzu traten noch weitere Ausdifferenzierungen wie die Spezialstahlherstellung oder die Gießereikunde. Gleichzeitig entstand mit der Einrichtung zusätzlicher Stellen die Eisenhüttenkunde "als hierarchisch geordnetes Statussystem" (ebd.: 39) - ein wichtiges Merkmal fortgeschrittener Disziplinbildung und notwendige Voraussetzung für die disziplinäre Selbstreproduktion, da der nächsten Wissenschaftlergeneration somit eine disziplinspezifische wissenschaftliche Laufbahn ermöglicht wurde. Ein weiterer wichtiger Aspekt der personellen Ausdifferenzierung war, dass dem einzelnen Lehrer nun mehr Zeit für seine Forschungstätigkeit zur Verfügung stand.

Mit der Studienreform einher ging ein starker Institutionalisierungsschub. Bereits während der beiden Unterrichtskonferenzen erhielt die Aachener Eisenhüttenkunde die Zusage, ihre reproduktiven und produktiven Institutionen ganz wesentlich ausbauen zu können. Die Zahl der etatmäßigen Stellen stieg von zwei auf sieben an und die der Assistentenstellen von zwei auf acht. Neben der Einrichtung zusätzlicher Professuren und Dozenturen wurde ferner ein modernes, 1,5 Millionen Mark teures Institut mit verschiedenen Lehr- und Forschungslaboratorien eingerichtet (Anonym 1906, 1910). Damit erreichte das Aachener Institut eine bauliche Ausstattung, die vergleichbar mit dem ersten chemischen Laboratorium der Berliner Universität ist.

Auf kognitiver Ebene war die Einführung der Metallographie der zentrale Schritt hin zu einem eigenständigen methodischen Fundament der Eisenhüttenkunde. Entwickelt im letzten Drittel des 19. Jahrhunderts und praktiziert insbesondere in der hüttenkundlichen Forschung in Frankreich und England, wurde die Aneignung der Metallographie durch die Aachener 
Eisenhüttenkunde zentral für die Ausbildung eines eigenen Denkstils. ${ }^{15}$ Der 1903 erfolgte Aufbau eines ersten metallographischen Laboratoriums - verbunden mit der Einrichtung einer entsprechenden Dozentur - war wesentlich für die frühe lehrhafte Vermittlung dieser Methode und die damit verbundene Befähigung der Studierenden und Absolventen, diese in ihre Forschungsarbeiten $\mathrm{zu}$ integrieren und als Hilfsmittel für die Produktion wissenschaftlicher Erkenntnis einzusetzen. Für Aachen kann die Eröffnung des metallographischen Laboratoriums als Zäsur der eisenhüttenkundlichen Forschungstätigkeit angesehen werden: Von den 57 Arbeiten der Bände 2-5 der Mitteilungen aus dem Eisenhüttenmännischen Institut benutzten 42 neben anderen Methoden auch metallographische Untersuchungen, was knapp 74\% entspricht (Wüst 1908-1913). ${ }^{16}$ Die Metallographie fand unabhängig davon Verwendung, ob es sich um Fragen der Gießereitechnik, der Herstellung von Spezialstählen oder der theoretischen Eisenhüttenkunde handelte.

Eng verbunden mit der Metallographie war die Ausbildung einer eigenen Terminologie, die sich beispielsweise in der fortschreitenden Benennung der Gefügebestandteile des Eisens und seiner Legierungen zeigte. Darüber hinaus adaptierte die Eisenhüttenkunde zahlreiche Begriffe und theoretische Ansätze der physikalischen Chemie sowie Grundverständnisse und Ideen der Thermodynamik - beispielsweise zu Wärme- und Stoffbilanzen. Die Begriffsbildung war eine notwendige Voraussetzung für die nach der Jahrhundertwende einsetzende und in Aachen forciert vorangetriebene Theoriebildung der Eisenhüttenkunde.

Insbesondere in der Präzisierung und Interpretation des Eisen-Kohlenstoff-Diagramms kulminierten die verschiedenen Konsolidierungsschritte: der Aufbau produktiver Institutionen, die lehrhafte Vermittlung neuer Methoden, ihre Anwendung in experimentellen Studien und die theoretische Deutung der Untersuchungsergebnisse. Dabei führte die Erforschung des Zustandsdiagramms zunächst konsequent weg von der nachträglichen Sichtung und Ordnung des Erfahrungswissens hin zu einem eigenen Erkenntnissystem der Eisenhüttenkunde. So schüttelte sie ihre theoretisch-methodische Rückständigkeit aus der langen Herausbildungsphase der Disziplin ab und etablierte sich in einem einzigen Jahrzehnt als weitgehend konsolidierte Technikwissenschaft (Buchheim 1984: 79).

Mit der erfolgreichen Studienreform erzielte Wüst einen Prestigegewinn, der sich symbolisch in den Verleihungen des 


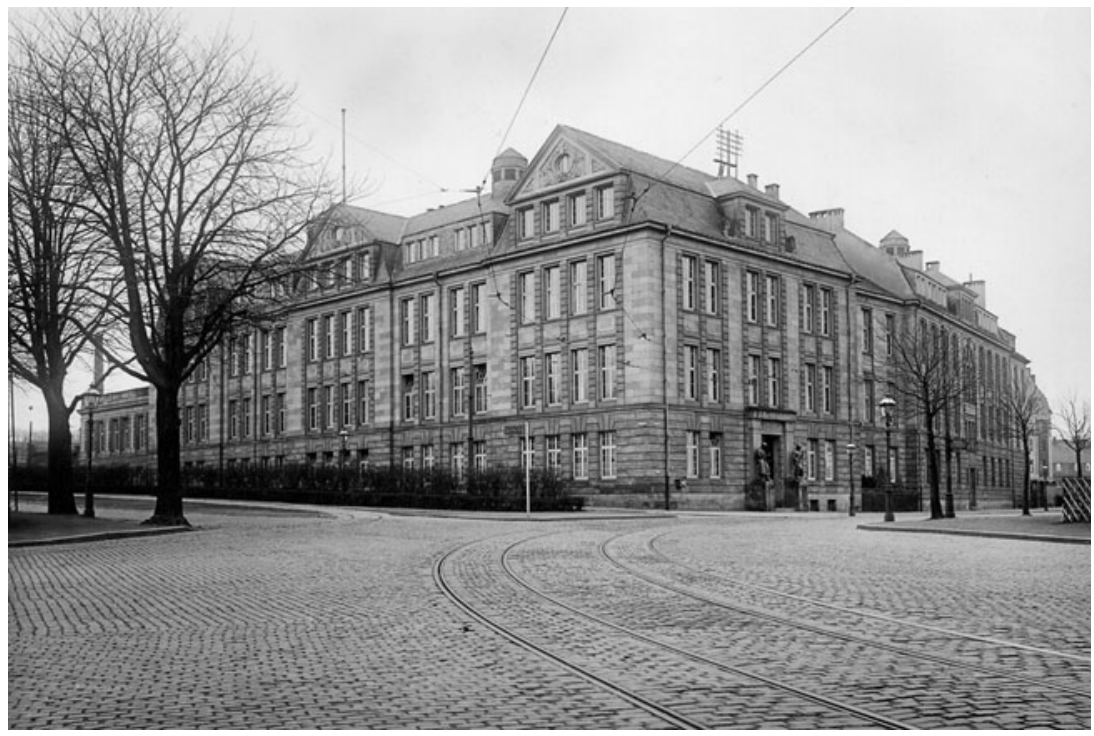

Roten Adlerordens 4. Klasse und des Titels eines Geheimen Abb. 3: Das neue Regierungsrates niederschlug. Sein Ansehen erreichte mit Eröff- Institut für das nung des neuen Instituts 1910 (Abb. 3) - rückgekoppelt an der Technischen dasjenige seines Instituts - einen vorläufigen Höhepunkt. Das Hochschule Aachen, Aachener Institut erhielt im Preußischen Abgeordnetenhaus O. D. (Hochschularchiv öffentlich die Zuschreibung des ersten eisenhüttenkundlichen Instituts Europas. ${ }^{17}$ Wüst wurde folgerichtig als erster Eisenhüttenkundler bezeichnet und bei der Einweihungsfeier mehrfach entsprechend geehrt: Ihm wurde unter anderem die Ehrendoktorwürde der Technischen Hochschule Stuttgart sowie die der Montanistischen Hochschule Leoben verliehen.

Die einzigartige Stellung des Aachener Instituts war damit allgemein anerkannt. Dies zeigte sich auch bei der Eröffnung der Breslauer Schwesterinstitution im folgenden Jahr. Sie war in ihrer Größe und Ausstattung mindestens ebenbürtig mit der Aachener Einrichtung und hatte gegenüber Aachen sogar den kleinen Vorteil, bereits über drei kleinere Spezialabteilungen zu verfügen: ein Aufbereitungslaboratorium, das mit Maschinen zur nassmechanischen und elektromagnetischen Trennung von Erzen ausgerüstet war, ein gastechnisches Laboratorium sowie ein Kokereilaboratorium (Die Redaction 1911). Aber die Breslauer Hochschule besaß noch keine eigene Reputation, und so unternahmen die dortigen Eisenhüttenkundler bei der Einweihungsfeier keine rhetorischen Versuche, den Aachenern den Rang streitig zu 
machen. Im Gegenteil priesen sie die Leistungen Fritz Wüsts und die Weltgeltung des Aachener Instituts (Anonym 1911).

Als Schlussstein der Konsolidierung kann die Durchsetzung des Deutungsmonopols der Eisenhüttenkunde im Streit zwischen der Zeitschrift des Vereins Deutscher Eisenhüttenleute, Stahl und Eisen, und der von Wüst mitherausgegebenen wissenschaftlichen Fachzeitschrift Metallurgie begriffen werden. In der nach einem heftigen Konflikt anerkannten Ausdifferenzierung der Kommunikationsmedien manifestierte sich der Bruch zwischen den beiden ehemals eng verbundenen Feldern der Eisenhüttenkunde und des Eisenhüttenwesens. Während des Streits boykottierten Wüst und seine Mitarbeiter Stahl und Eisen, indem sie dort keine wissenschaftlichen Arbeiten mehr veröffentlichten, was den Ruf der Zeitschrift schädigte. Auf der anderen Seite initiierte der Verein Deutscher Eisenhüttenleute einen Anzeigenboykott gegen die Metallurgie und beharrte vehement auf dem Vorrecht, alle wissenschaftlichen Arbeiten des Aachener Instituts in der Vereinszeitschrift veröffentlichen zu dürfen. Stahl und Eisen sollte also das bislang als einzige monothematische Fachzeitschrift für das Eisenhüttenwesen eingenommene Konsekrationsmonopol für das eisenhüttenkundliche Feld behalten. ${ }^{18}$ Nach einem fast vier Jahre andauernden Konflikt musste der Verein schließlich 1909 nachgeben (vgl. Krebs 2009: 388-397). Die Anerkennung des Existenzrechts einer der Theoriebildung und Grundlagenforschung gewidmeten Zeitschrift, die sich der Kontrolle der Stahlindustrie entzog, beschleunigte zugleich die weitere Entwicklung. Zentraler Forschungsgegenstand des Aachener Instituts war in dieser Zeit das Zustandsdiagramm Eisen-Kohlenstoff (Wüst 1909).

Noch vor Ausbruch des Ersten Weltkriegs erreichte die Aachener Eisenhüttenkunde damit die vollständige Ausbildung einer eigenen disziplinären Grundlage, auf deren Basis die weitere Entwicklung der Disziplin stattfinden konnte (vgl. Krebs 2007, 2009). Die disziplinäre Ausprägung der Wüst'schen Schule diente dem Kultusministerium zudem als Vorbild für die anderen Studienstandorte - diesen wurde unter anderem die Übernahme des Aachener Curriculums empfohlen. ${ }^{19}$

\section{Stadiale Gliederung der eisenhüttenkundlichen Dis- ziplingenese}

Die Untersuchung der Disziplingenese der Eisenhüttenkunde am Beispiel der Technischen Hochschule Aachen hat gezeigt, dass 
diese sich geradezu paradigmatisch dem Dresdener Periodisierungsmodell entsprechend entwickelt hat: Auf allen drei Analyseebenen - Gegenstandsorientierung, Institutionalisierung und Selbstreproduktion - haben zwischen 1870 und 1914 qualitative Entwicklungen stattgefunden, wie sie innerhalb des Dresdener Konzepts beschrieben werden. Während der Amtszeit von Friedrich Dürre lag der Schwerpunkt der disziplinären Tätigkeit auf der lehrhaften Vermittlung des geordneten und systematisierten Erfahrungswissens. Die institutionelle Basis der Hüttenkunde war insbesondere in Aachen nur schwach entwickelt und zudem auf die reproduktive Aufgabe des Fachs beschränkt. An dieser Stelle bleibt festzuhalten, dass die akademische Institutionalisierung der Eisenhüttenkunde der kognitiven Verwissenschaftlichung der Disziplin um Jahrzehnte voranging.

Der Wechsel der etatmäßigen Professur für Eisenhüttenkunde von Dürre zu Wüst 1901 kann rückblickend als tiefgreifende Zäsur und Initialzündung für die Konsolidierung dieses disziplinären Feldes angesehen werden. Die Änderung der Gegenstandsorientierung, wie sie im neuen Curriculum sichtbar wurde ${ }^{20}$, und der Auf- und Ausbau des institutionellen Rahmens der Hüttenkunde zielten auf die Ausdifferenzierung und damit Spezialisierung des eisenhüttenkundlichen Studiums und zugleich auf eine inhaltlichmethodische Emanzipation von der anorganischen und analytischen Chemie. Hierzu inkorporierte die Eisenhüttenkunde verstärkt neue Methoden der physikalischen Chemie. Die Verbreiterung der institutionellen Basis durch die Einrichtung weiterer Personalstellen und der Aufbau neuer, mit den modernsten Einrichtungen ausgestatteter Laboratorien zielten auf die Verwissenschaftlichung der Disziplin und bedingten einander wechselseitig.

Mit dem erweiterten Laboratoriumsbetrieb wurde zugleich die Möglichkeit für eine disziplinspezifische Laufbahn geschaffen, und nachdem ihm die ersten eigenen Absolventen zur Verfügung standen, griff Wüst dann auch vornehmlich auf sie zurück und platzierte sie auf aussichtsreichen Stellen. Seinen Schüler Paul Oberhoffer installierte er 1910 als Dozent für Metallographie und Werkstoffkunde an der neugegründeten Technischen Hochschule Breslau. Im Dezember 1917 entschied sich dann die Abteilung IV der Aachener Hochschule für Oberhoffer als Nachfolger von Wüst, der - Zeichen seiner dominierenden Position auf dem eisenhüttenkundlichen Feld - zum Gründungsdirektor des KaiserWilhelm-Instituts für Eisenforschung ernannt worden war (vgl. 
Maier 2007, Flachowsky 2007). ${ }^{21}$ Mit Oberhoffers Berufung und seinem endgültigen Wechsel nach Aachen 1921 fand die Selbstreproduktion der Wüst'schen Schule ihren vorläufigen Abschluss (vgl. Krebs 2009: 307f.).

Eine weitere zentrale Wegmarke für die Konsolidierung der Eisenhüttenkunde war die Etablierung eigener kommunikativer Institutionen durch die Begründung einer der wissenschaftlichen Eisenhüttenkunde vorbehaltenen Fachzeitschrift, der Metallurgie. Als neue Leitwährung setzten sich Originalbeiträge durch, in denen die veränderten Erkenntnisobjekte und -methoden behandelt wurden. Hierdurch erfolgte zum einen eine Abkopplung der eisenhüttenkundlichen Forschung von der betrieblichen Praxis, da vornehmlich Grundlagenforschung betrieben wurde, und zum anderen änderten sich hierdurch die Publikationsstrategien. Die konfliktgeladene Durchsetzung des Deutungs- und Publikationsmonopols der von Wilhelm Borchers ${ }^{22}$ und Wüst herausgegebenen Zeitschrift Metallurgie zog zudem eine neue Grenze zwischen Wissenschaft und Praxis (Bourdieu 2001: 353).

Der gesamte Konsolidierungsprozess kulminierte im Neubau des eisenhüttenkundlichen Instituts, das bereits zeitgenössisch als Meilenstein der Disziplinentwicklung und Vorbild für die anderen eisenhüttenkundlichen Institute galt. Das Breslauer Institut, das ein Jahr später eingeweiht wurde, war ausdrücklich mit Rückgriff auf die Expertise der Aachener Eisenhüttenkundler geplant worden (Anonym 1911). Die verschiedenen Einrichtungen des Aachener Instituts und die sich etablierende institutionelle Basis des neuen Forschungsprogramms bildeten die Synthese zu einem relativ geschlossenen System eisenhüttenkundlicher wissenschaftlicher Tätigkeiten mit einem spezifischen Korpus gemeinsamer Problemstellungen, Methoden und Begriffe. Dazu gehörten vor allem die Einführung und Weiterentwicklung der Metallographie in Forschung und Lehre sowie die Präzisierung und Interpretation des Eisen-Kohlenstoff-Diagramms.

Ein weiterer Befund zur stadialen Gliederung der Disziplingenese der Eisenhüttenkunde ist, dass sie im Gegensatz zu anderen klassischen Technikwissenschaften erst spät in ihre Konsolidierungsphase eintrat, so gegenüber dem Maschinenbau fast drei Jahrzehnte später (Hänseroth/Mauersberger 1998: 229-233). Dafür verlief die Konsolidierung wesentlich schneller, so dass die Disziplingenese dann innerhalb eines Jahrzehnts abgeschlossen war. 


\section{Zur sozialen Praxis der Technikwissenschaften}

Unter der vorgeschlagenen Betrachtungsweise können die vom Dresdener Konzept beschriebenen Konsolidierungsschritte als notwendige Bedingungen der Verwissenschaftlichung der Eisenhüttenkunde aufgefasst werden. Demgegenüber muss die Autonomisierung als hinreichende Bedingung ihrer Verwissenschaftlichung begriffen werden. Das würde bedeuten, dass die Verwissenschaftlichung der Eisenhüttenkunde nur unter Durchsetzung ihres Deutungsmonopols erfolgen konnte, wobei die dazu notwendige Brechungsstärke der Disziplin an ihre Kapitalausstattung gekoppelt war.

$\mathrm{Zu}$ berücksichtigen ist dabei die Besonderheit der Eisenhüttenkunde als einer technikwissenschaftlichen Disziplin. Sie beruht auf der untrennbaren Verknüpfung der Technikwissenschaften mit dem ökonomischen Feld, während Autonomie für Bourdieu konstitutiv für das wissenschaftliche Feld ist. Diese paradox anmutende Eigenart konsolidierter Technikwissenschaften soll abschließend noch einmal genauer betrachtet werden.

Bourdieus Konzeption des wissenschaftlichen Feldes schreibt diesem eine Anordnung entlang zweier Pole zu: dem heteronomen Gesellschaftspol und dem autonomen Wissenschaftspol. Am heteronomen Pol befinden sich Disziplinen wie Jura und Medizin, am autonomen Pol beispielsweise die Naturwissenschaften (Bourdieu 1992: 107). Die Technikwissenschaften dürften dem gesellschaftlichen Pol zugeordnet sein, wobei dieser präziser als wirtschaftlicher Pol zu charakterisieren wäre. ${ }^{23}$ In sich weisen alle Disziplinen wiederum dieselbe Struktur auf, das heißt, die einzelnen Wissenschaftler können sich innerhalb des sozialen Raums ihrer Disziplin entweder dem einen oder dem anderen Pol zuwenden.

Für Bourdieu liegt der grundlegende Nomos moderner Wissenschaften in ihrer Unabhängigkeit von wirtschaftlichen und politischen Machtinstanzen. Die im Umfeld des Dresdener Forschungsprogramms entstandenen Untersuchungen der Disziplingenese verschiedener Technikwissenschaften haben gezeigt, dass diese in ihrer Herausbildungsphase nur über einen heteronomen Pol verfügten. Dies entspricht in dieser Zeit ihrer ausschließlichen Orientierung auf die technische Praxis. Dafür steht auch die anfängliche Zuständigkeit des preußischen Handelsministeriums für die Bergakademien und polytechnischen Schulen sowie die Beschränkung der wissenschaftlichen Erkenntnistätigkeit auf die 
Ordnung und Systematisierung des praktischen Erfahrungswissens. Erst mit der Konsolidierung einzelner technikwissenschaftlicher Disziplinen bildeten diese einen autonomen Pol aus. ${ }^{24}$

Für die Eisenhüttenkunde hat der Streit zwischen Friedrich Dürre und Joseph Schlink anschaulich gezeigt, dass die Akteure des ökonomischen Felds in den 1870er Jahren noch eine Position einnahmen, die es ihnen erlaubte, die Ausbildung eines autonomen Pols $\mathrm{zu}$ verhindern. Paradoxerweise begünstigte die Dominanz des heteronomen Pols der Eisenhüttenkunde, die bis zur Jahrhundertwende fortbestand, die Durchsetzung der von Fritz Wüst konzipierten Studienreform. Den Vertretern der Eisenund Stahlindustrie wurde das Recht zugebilligt, weitgehend über die Erkenntnisobjekte und -methoden der Eisenhüttenkunde zu entscheiden.

Der bestimmende Einfluss des Vereins Deutscher Eisenhüttenleute auf die Studienreform kann noch als typisches Merkmal der Herausbildungsphase gedeutet werden. In dieser Zeit war der heteronome Pol des eisenhüttenkundlichen Feldes noch stark ausgeprägt, und ,[j]e heteronomer [...] ein Feld, desto unvollständiger ist dort der Wettbewerb und desto leichter fällt es den Akteuren, äußere Mächte in die wissenschaftlichen Kämpfe einzuschleusen" (Bourdieu 1998: 28). Das heißt, Wüst konnte den Wettbewerb zwischen den eisenhüttenkundlichen Instituten gerade und nur mithilfe der Vertreter der Eisen- und Stahlindustrie außer Kraft setzen und einseitig für sich entscheiden. Andererseits war Wüst nicht gewillt, seine wissenschaftliche Arbeit vonseiten der Industriellen sanktionieren $\mathrm{zu}$ lassen. Vielmehr strebte er danach, das eisenhüttenkundliche Feld aus der Umklammerung durch die hüttenmännische Praxis zu lösen. Das heißt, es zu autonomisieren, denn ,[j]e autonomer [...] ein Feld ist, je näher also an einem reinen und vollständigen Wettbewerb, desto eher ist dort die Zensur eine rein wissenschaftliche, die rein gesellschaftliche Eingriffe [...] ausschließt" (ebd.: 28). Während Wüst also anfangs die Macht des Vereins innerhalb des wissenschaftlichen Feldes für sich einzusetzen wusste, setzte er nach Abschluss der Studienreform und der damit einhergehenden Saturation seiner Ressourcenansprüche alles daran, diese Macht selbst zu brechen. Er kämpfte vehement für eine Ausbildung des autonomen Pols der Eisenhüttenkunde und wandte sich gegen den Einfluss des Vereins auf das wissenschaftliche Feld - einen Einfluss, der mit Bourdieu als Tyrannei charakterisiert werden kann: 
Tyrannei herrscht beispielsweise, wenn die politische Macht oder die wirtschaftliche Macht in das Feld der Wissenschaft oder das der Literatur eingreift, sei es unmittelbar, sei es vermittels einer spezifischeren Macht wie der von Akademien, Verlegern, Kommissionen oder der des Journalismus [...], um dort ihre eigenen Hierarchien zu errichten und die Selbstbehauptung spezifischer Hierarchisierungsprinzipien zu unterdrücken. (Bourdieu 2001b: 131f.)

Die Autonomisierung war jedoch keine einseitige Setzung Wüsts, sondern bildete sich erst durch eine Reihe symbolischer Kämpfe um die Deutungsmacht der wissenschaftlichen Eisenhüttenkunde heraus. Neben dem Streit um die Zukunft des Thomasverfahrens (Krebs 2007) kam dem Kampf um das Existenzrecht der Metallurgie besondere Bedeutung zu: eine der wissenschaftlichen Eisenhüttenkunde gewidmete Zeitschrift, die sich der Kontrolle durch die Stahlindustrie entzog. Der Verein Deutscher Eisenhüttenleute kämpfte vehement darum, sein Publikations- und Konsekrationsmonopol und damit Stahl und Eisen als gemeinsame Plattform von Wissenschaft und Praxis zu erhalten. Wüst hingegen riskierte den Verlust seines sozialen Kapitals, um die Metallurgie als unabhängige wissenschaftliche Zeitschrift zu etablieren. Peter Weingart bezeichnet die Zeitschriftengründung auch als den wichtigsten Institutionalisierungsschritt einer wissenschaftlichen Disziplin:

Eine Gruppe, die eine Zeitschrift oder eine wissenschaftliche Gesellschaft gründet, stellt sich damit gleichsam außerhalb des geltenden Rechts - sie lehnt die geltenden Qualitäts- und Auswahlkriterien ab. [...] Die Gründung einer Zeitschrift und/oder einer wissenschaftlichen Gesellschaft bedeutet die institutionelle Verfestigung und Verselbständigung eines Satzes von Orientierungskomplexen [...] in Form eines paradigmatischen Programms, das zumindest partiell zu etablierten Orientierungskomplexen in Konflikt steht. (Weingart 1974: 30, 32)

Mit der Konsolidierung der Eisenhüttenkunde und der Etablierung ihres wissenschaftlichen Deutungsmonopols war zugleich die Ausbildung der chiastischen Struktur des eisenhüttenkundlichen Feldes verbunden. ${ }^{25}$ Das heißt, dass erst mit der disziplinären Theoriebildung wissenschaftliche Tätigkeit um ihrer selbst willen erfolgen konnte, eisenhüttenkundliche Grundlagenforschung also als relevant und prestigeträchtig anerkannt wurde. Dies erklärt, warum Wüst nach der Studienreform auf einen Teil seines zuvor benötigten sozialen Kapitals verzichten konnte, denn unter den konsolidierten Produktionsbedingungen benötigte er dies zur Akkumulation wissenschaftlichen Kapitals - als nunmehr im Sinne Bourdieus einzige legitime Kapitalform ${ }^{26}$ - nicht mehr.

Hieraus folgt außerdem, dass das ökonomische und soziale Kapital des Vereins Deutscher Eisenhüttenleute an Wert verlor, da 
die Eisenhüttenkundler nur von den anderen Mitgliedern der Scientific Community im engeren Sinne Anerkennung und damit wissenschaftliches Kapital für ihre Forschungsleistungen erhalten konnten: Im Sinne Ludwik Flecks verschob sich mit der Konsolidierung die Grenze zwischen dem kleineren esoterischen Kreis der Wissenschaftler und dem größeren exoterischen Kreis der Eisenhütteningenieure (Fleck 1980: 138f.). Die beherrschende Machtstellung der Aachener Eisenhüttenkunde transformierte demnach die Struktur des gesamten eisenhüttenkundlichen Feldes und brachte den anderen Eisenhüttenkundlern ebenfalls wissenschaftliche Gewinne ein, insofern sie sich den neuen Regeln entsprechend ins Spiel einbrachten - was sie, wie der Erfolg der Zeitschrift Metallurgie zeigte, auch taten. Das Autorenverzeichnis der Metallurgie war damit Ausweis wissenschaftlicher Macht und zugleich symbolisches Kapital, das Wüst und sein Kollege Borchers gegen Stahl und Eisen ins Feld führen konnten. Hieran zeigt sich deutlich, dass die Aachener Hüttenkundler durch ihre Einsätze und Strategien eine beherrschende Position im Feld erlangt hatten, die es ihnen ermöglichte, die Regeln der Disziplin zu ihren Gunsten zu ändern.

Das Spezifische der Technikwissenschaften liegt nun darin, dass sie zwei Abnehmergruppen aufweisen: zum einen die anderen Technikwissenschaftler und zum anderen die Vertreter der technisch-industriellen Praxis. Technikwissenschaftliches Wissen ist immer auch Mittel für erfolgreiches technisches Gestalten: „Die Technikwissenschaften sind nicht nur für die Theorie da, sondern für die Praxis. " (Banse u. a. 2006) Hier ist die auch in der Disziplin selbst geführte Debatte über den Wissenschaftstyp der Eisenhüttenkunde einzuordnen: Ist sie angewandte Naturwissenschaft oder eventuell auch gar keine Wissenschaft (Schenck 1971: 24, Banse u. a. 2006)? Zum anderen erklärt sich aus dieser besonderen Struktur des technikwissenschaftlichen Felds die Schwierigkeit, einen eigenen autonomen Pol auszubilden. Wie lässt sich Autonomie erreichen, wenn die wissenschaftliche Tätigkeit auf den heteronomen Pol des Feldes zielt, und warum sollten die Technikwissenschaften überhaupt nach Autonomie streben?

Dieses Streben ist für Bourdieu der Struktur des wissenschaftlichen Feldes inhärent. Die Sublimation äußerer Zwänge liegt demnach in der besonderen Form der Illusio beschlossen, die zur Teilhabe am Feld notwendig dazugehört: der Wissenschaftsglaube, eine Art interesseloses Interesse und das Interesse an der Interesselosigkeit (Bourdieu 1998: 27). 
Das Fallbeispiel der Eisenhüttenkunde hat gezeigt, dass es den Aachener Akteuren vor allem darum ging, eigenständig über die sozialen und kognitiven Belange der Disziplin entscheiden zu können, also ein von den Eigeninteressen der Stahlindustrie und dem Einfluss anderer wissenschaftlicher Disziplinen unabhängiges Deutungssystem zu etablieren. Soweit erklärt sich das Eigeninteresse der Eisenhüttenkundler an der Durchsetzung disziplinärer Autonomie. Bei der Deutung des Interesses der industriellen Praxis an der Autonomie der Technikwissenschaften helfen Bourdieus Überlegungen zur Machtausübung in modernen ausdifferenzierten Gesellschaften. Er schreibt:

\footnotetext{
Sie wird auf unsichtbare und anonyme Weise durch scheinbar anarchische, in Wirklichkeit aber strukturell zwingende Aktionen und Reaktionen von Akteuren und Institutionen ausgeübt, die in miteinander konkurrierende und einander zugleich ergänzende Felder wie zum Beispiel in das der Ökonomie und das der Bildung integriert sind und in immer längeren und komplexeren, also symbolisch auch immer effizienteren, aber zumindest potentiell auch immer mehr Macht- und Autoritätskonflikten Raum gebenden Kreisläufen gegenseitigen Legitimierens fungieren. (Bourdieu 2001b: 130)
}

Die hierin beschriebenen Kreisläufe der Legitimation setzen voraus, dass die einzelnen miteinander verbundenen Felder über ein gewisses Maß an Autonomie verfügen, denn wirklich effiziente gegenseitige symbolische Dienste sind nur unter der Bedingung möglich, dass die einzelnen Akteure das Recht auf unabhängige Urteile haben, das selbst ,die Möglichkeit kritischer Infragestellung zulässt" (ebd.: 133). Das heißt, dass die autonome Eisenhüttenkunde der Stahlindustrie effektivere symbolische Dienste leisten konnte, als sie dies ohne ihr Recht zur kritischen Infragestellung hätte tun können. Ein Beispiel ist der Streit zwischen den Schienenproduzenten und der staatlichen Eisenbahnverwaltung um die Qualität des Eisenbahnobermaterials: Hier konnte die deutsche Eisenhüttenkunde zugunsten der Thomasschiene und damit für die ökonomischen Investitionen der deutschen Stahlindustrie Stellung beziehen. Andererseits musste die Stahlindustrie ein kritisches Qualitätsurteil fürchten, was der Eisenhüttenkunde zusätzliche symbolische Macht verlieh (Krebs 2007). In den Vereinigten Staaten und Großbritannien wurde dagegen nach einer Reihe von Eisenbahnunglücken die Verwendung von Thomasstahl zur Schienenherstellung untersagt (Aldrich 1999).

Die Autonomie eines Feldes muss allerdings nicht vollkommen sein und kann dies im Fall der Technikwissenschaften auch nicht sein. Stattdessen ist von einer relativen Autonomie der technikwissenschaftlichen Felder auszugehen, die unmittelbare ökonomische Zwänge aufhebt und eine unabhängige wissen- 
schaftliche Tätigkeit garantiert, aber die mittelbare Abhängigkeit der Technikwissenschaften von der technischen Praxis niemals ganz überwindet. Der Autonomisierungsprozess ist zudem nicht irreversibel, sondern die beiden Pole des wissenschaftlichen Feldes können im Laufe der Zeit unterschiedlich stark ausgebildet sein. Von der Ausprägung der autonomen Seite hängt aber die Brechungsstärke wissenschaftlicher Disziplinen ab. Das bedeutet, ohne ein ausreichendes Maß Autonomie nehmen externe Einflüsse anderer Felder zu, worunter zugleich der Nomos der Wissenschaft leidet und in letzter Konsequenz Wissenschaft aufhört, Wissenschaft zu sein.

Vor dem Hintergrund dieser theoretischen Überlegungen lässt sich die Konsolidierung der Aachener Eisenhüttenkunde als symbolischer Kampf zwischen Fritz Wüst und den Vertretern der Eisen- und Stahlindustrie begreifen, der zur Konstruktion eines „Systems der Unterschiede” führte. Darin akzeptierten die Wissenschaftler es, Wissenschaftler und nicht Unternehmer zu sein, und die Unternehmer respektierten es, Unternehmer zu sein und verzichteten darauf, Wissenschaftler zu werden (Bourdieu 1991: 71). Der „tyrannische“ Einfluss der Industriellen auf die Erkenntnisinteressen und -ziele der Eisenhüttenkunde wurde so aufgehoben. Erst damit war die Verwissenschaftlichung der Disziplin möglich geworden.

Die Disziplingenese der Eisenhüttenkunde zeigt anschaulich, dass ihre Entwicklung nicht als eine Art Parthenogenese begriffen werden kann, als Prozess, aus dem sich die Wissenschaft gewissermaßen selbst hervorbringt. Diese teleologische Annahme, die dem Dresdener Konzept als Teil der marxistischen Geschichtsschreibung inhärent ist, schmälert dagegen nicht die Beschreibungskraft des Entwicklungsmodells. Das Dresdener Konzept ist wiederum hilfreich, um die Theorie der Praxis für die Geschichte der Technikwissenschaften fruchtbar zu machen. Während Bourdieu selbst nicht über die Technikwissenschaften gearbeitet hat, finden sich in den Dresdener Studien Strukturmerkmale beschrieben, die die Einordnung und Kontextualisierung der Quellen zur Aachener Hochschulgeschichte ermöglichen.

Bourdieus Theorie der Praxis vermag die sozialen Triebkräfte des wissenschaftlichen Wandels aufzuzeigen. Mithilfe seiner theoretischen Annahmen, speziell zur Struktur des wissenschaftlichen Feldes, lässt sich den Quellen ihr sozialer Sinn entlocken. Es gelingt auf diese Weise, kontextuelle Zusammenhänge aufzudecken, das Agieren in sozialen Handlungsfeldern verständlich zu 
machen und den Einfluss einzelner Akteure herauszuarbeiten. Schließlich veranschaulicht die Geschichte der Eisenhüttenkunde die von Bourdieu selbst beschriebene Eigenart wissenschaftlicher Felder: Nur hier können die Akteure die Spielregeln selbst ins Spiel bringen und, wie im Fall Fritz Wüsts, zu ihren Gunsten verändern (Bourdieu 1998: 25).

\section{Nachweis}

Der Beitrag ist eine erweiterte Fassung meiner Disputation, die am 12. Februar 2008 an der RWTH Aachen stattfand. Der Titel spielt auf Pierre Bourdieus Studie Die Regeln der Kunst (2001a) an. Zur Druckfassung der Dissertation siehe Krebs 2009.

Open Access This article is distributed under the terms of the Creative Commons Attribution Noncommercial License which permits any noncommercial use, distribution, and reproduction in any medium, provided the original author(s) and source are credited.

\section{Anmerkungen}

1 Biographische Angaben über Fritz Wüst (1860-1938) finden sich im Rheinisch-westfälischen Wirtschaftsarchiv, Köln, Abt. 130, 3001030/1, Programm und Jahresbericht der Kgl. Preussischen Maschinenbau- und Hüttenschule in Duisburg, Duisburg 1901, und in Goerens/Körber/ Petersen 1938.

2 Geheimes Staatsarchiv Preußischer Kulturbesitz, Berlin (zukünftig GSTA PK), I. HA Rep. 151 Finanzministerium, IC 6967, Haus der Abgeordneten, Protokoll der Sitzung vom 29. April 1910.

3 Historisches Archiv des Vereins Deutscher Eisenhüttenleute, Düsseldorf (im Folgenden HA VDEh), Se 12 bis, Stenographische Niederschrift der Besprechung über Hochschulausbildung vom 31. Mai 1926, S. 5.

4 Ernst Friedrich Dürre (1834-1905) hatte zuvor seit 1865 als Assistent bei Hermann Wedding an der Berliner Bergakademie gearbeitet. Vgl. zu seiner Biographie GSTA PK, I. HA Rep. 76 Kultusministerium, Vb, Sekt. 6, Tit. II, Nr. 1 Bd. I, Nachweisung über die persönlichen und dienstlichen Verhältnisse des Professors Dr. Dürre, o. D. (Mai 1886).

5 Dagegen verfügte der Aachener Vertreter der anorganischen Chemie sogar über ein eigenes, Forschungszwecken vorbehaltenes Privatlaboratorium im Neubau des Chemischen Instituts (TH Aachen 1879).

6 Dürre besuchte u. a. die Pariser École des Mines, die Londoner Royal School of Mines und die Lütticher École des arts et manufactures et des mines.

7 Der erste Vorsitzende des Technischen Vereins für das Eisenhüttenwesen war der Industrielle Leopold Hoesch. Ihm folgten Carl Lueg (1879-1905), Direktor der Firma Jacobi, Haniel \& Huyssen in Sterkrade, der späteren 
Gutehoffnungshütte, und Friedrich Springorum (1905-1917), Direktor des Eisen- und Stahlwerks Hoesch in Dortmund (Anonym 1935: 1258, 1265, 1347f., 1362).

8 Hochschularchiv RWTH Aachen (im Folgenden HAAc), 934b, F. Wüst und W. Borchers an den Kultusminister vom 2. November 1907. Vgl. auch Chezeau 2004: 103f., 199f.

9 HA VDEh, Se 12 bis a-b, Eingabe des VDEh an Handelsminister Möller vom 14. Mai 1902; ebd., Eingabe des VDEh an Kultusminister Studt vom 14. Mai 1902.

10 Vgl. HAAc, 934a, Fritz Wüst: Das Studium des Eisenhüttenwesens und die Errichtung eines neuen eisenhüttenmännischen Instituts an der Königlichen Technischen Hochschule zu Aachen (Sonderdruck); HA VDEh, Se 12 bis $a-b$, Verhandlungen über den Ausbau des Unterrichts im Eisenhüttenwesen an den Technischen Hochschulen und Bergakademien Preußens, Besprechung vom 7. November 1903, Berlin 1903; ebd., Verhandlungen über den Ausbau des Unterrichts im Eisenhüttenwesen an den Technischen Hochschulen und Bergakademien Preußens, Besprechung vom 8. Januar 1904, Berlin 1904.

11 Vgl. dazu die Dankesschreiben: HA VDEh, Ag 10 Bd. I, E. Schrödter an F. Wüst vom 19. Januar 1903; ebd., C. Lueg und E. Schrödter an Rektor TH Aachen vom 21. Januar 1903.

12 Friedrich Springorum wurde zudem 1905 zum neuen Vorsitzenden des VDEh gewählt und nahm damit eine beherrschende Stellung im ökonomischen Feld ein.

13 Historisches Archiv Hoesch (zukünftig HoeschA), Dortmund, F4a55, F. Springorum an E. Schrödter vom 2. März 1905.

14 Vgl. die Berliner Verhandlungsprotokolle im HA VDEh, wie Anm. 11.

15 Der Begriff des Denkstils, als disziplinierte, gemeinsame Stimmung wissenschaftlichen Denkens, erscheint passend, um die Herausbildung einer Wüst'schen Denkschule zu beschreiben (Fleck 1980: 187-190).

16 Der erste Band ist insofern ein Sonderfall, da die ersten darin veröffentlichten Arbeiten noch vor der Einrichtung des metallographischen Laboratoriums entstanden waren.

17 GSTA PK, I. HA Rep. 151 Finanzministerium, IC 6967, Haus der Abgeordneten, Protokoll der Sitzung vom 29. April 1910.

18 Die Zeitschrift Metallurgie, 1912 umbenannt in Ferrum, stellte 1917 ihr Erscheinen ein. Das daraus entstandene Vakuum nutzte 1927 der VDEh, um gemeinsam mit dem Kaiser-Wilhelm-Institut für Eisenforschung das Archiv für das Eisenhüttenwesen herauszugeben: ein Stahl und Eisen ergänzendes Periodikum, das Raum für Originalarbeiten bot und zugleich in der (Teil-)Zuständigkeit des Vereins verblieb.

19 HoeschA, F4a39, Aktennotiz E. Schrödter, o. D. (zur Unterredung mit O. Naumann am 21. Januar 1907).

20 Die Verschiebung fand von der chemisch-metallurgischen zur maschinentechnischen Seite der Hüttenkunde statt - Wüst selbst bezeichnete dies 1926 als einen seiner größten Fehler (HA VDEh, Se 12 bis, Stenographische Niederschrift der Besprechung über Hochschulausbildung vom 31. Mai 1926, S. 13 f.).

21 Die Darstellung der Gründungsgeschichte gerät leider etwas zu kurz, wenn z. B. die erzwungene Demission Fritz Wüsts im Jahre 1922 schlicht als Eintritt in den Ruhestand bezeichnet wird (Flachowsky 2007: 160).

22 Biographische Angaben zu Wilhelm Borchers (1856-1925) finden sich bei Röntgen 1955.

23 Bourdieu selbst äußert sich leider nicht explizit zu den Technikwissenschaften; das Register des Homo academicus (Bourdieu 1992) führt weder die Begriffe „Ingenieur“ noch „Technik“ auf. Vgl. dazu auch Felt u. a. 1995: 78. 
24 Ein bekanntes und intensiv untersuchtes Beispiel ist der Streit um die Verwissenschaftlichung der Maschinenbaukunde (Richter 1984). Speziell zum Streit zwischen Franz Reuleaux und Alois Riedler vgl. König 1999, Heymann 2005, Manegold 1990.

25 Unter der chiastischen Struktur des wissenschaftlichen Feldes versteht Bourdieu die kreuzweise angeordneten Pole der Autonomie bzw. Heteronomie. Auf der autonomen Seite befinden sich die Träger symbolischen Kapitals im Sinne wissenschaftlicher Anerkennung. Der heteronome Pol wird dagegen von Trägern universitärer Macht bestimmt, die über materielle Ressourcen und institutionelle Positionen verfügen (Bourdieu 1998: 27, 2001a: 189).

26 Bourdieu unterscheidet zwei Sorten wissenschaftlichen Kapitals: symbolisches Kapital, das auf wissenschaftlichen Meriten beruht, und universitäres Kapital, das auf Verwaltungsmacht aufbaut (Bourdieu 1998: 31).

\section{Literatur}

Aldrich, Mark, 1999. „The Peril of the Broken Rail”. The Carriers, the Steel Companies, and Rail Technology, 1900-1945. Technology and Culture, 40, 263-291.

Anonym, 1887. Rezension E. F. Dürre. Die Anlage und der Betrieb der Eisenhütten. Stahl und Eisen, 7, 372-373.

Anonym, 1892. Rezension E. F. Dürre. Die Anlage und der Betrieb der Eisenhütten. Zeitschrift des VDI, 36, 967.

Anonym, 1906. Institut für das gesamte Hüttenwesen in Aachen. Stahl und Eisen, 26, 806-809.

Anonym, 1910. Einweihung der Institute für Hüttenkunde zu Aachen. Stahl und Eisen, 30, 1081-1085.

Anonym, 1911. Vereins-Nachrichten. Verein Deutscher Eisenhüttenleute. Stahl und Eisen, 31, 1608-1611/1650-1651.

Anonym, 1935. 1860 bis 1935. 75 Jahre Verein Deutscher Eisenhüttenleute. Stahl und Eisen, 55, 1253-1450.

Ash, Mitchell G., 2002. Wissenschaften und Politik als Ressourcen für einander. In: Rüdiger vom Bruch und Brigitte Kaderas, Hg., Wissenschaften und Wissenschaftspolitik. Stuttgart: Franz Steiner, 32-51.

Banse, Gerhard/Grunwald, Armin/König, Wolfgang/Ropohl, Günter, Hg., 2006. Erkennen und Gestalten. Eine Theorie der Technikwissenschaften. Berlin: Edition Sigma.

Bourdieu, Pierre, 1983. Ökonomisches Kapital, kulturelles Kapital, soziales Kapital. In: Reinhard Kreckel, Hg., Soziale Ungleichheiten. Göttingen: Schwartz, 183198.

Bourdieu, Pierre, 1987. Die feinen Unterschiede. Kritik der gesellschaftlichen Urteilskraft. Frankfurt a. M.: Suhrkamp.

Bourdieu, Pierre, 1991. Die Intellektuellen und die Macht. Hamburg: Vsa.

Bourdieu, Pierre, 1992. Homo academicus. Frankfurt a. M.: Suhrkamp.

Bourdieu, Pierre, 1998. Vom Gebrauch der Wissenschaft. Für eine klinische Soziologie des wissenschaftlichen Feldes. Konstanz: UVK.

Bourdieu, Pierre, 2001a. Die Regeln der Kunst. Genese und Struktur des literarischen Feldes. Frankfurt a. M.: Suhrkamp.

Bourdieu, Pierre, 2001b. Meditationen. Zur Kritik der scholastischen Vernunft. Frankfurt a. M.: Suhrkamp.

Buchheim, Gisela, 1984. Zu einigen Fragen der Periodisierung der Technikwissenschaften. Dresdener Beiträge zur Geschichte der Technikwissenschaften, 9, $72-83$. 
Champagne, Patrick, 1998. Vorwort. In: Pierre Bourdieu, Vom Gebrauch der Wissenschaft. Für eine klinische Soziologie des wissenschaftlichen Feldes. Konstanz: UVK, 7-14.

Chezeau, Nicole, 2004. De la Forge au Laboratoire. Naissance de la métallurgie physique 1860-1914. Rennes: Presses Universitaires de Rennes.

Die Redaction, 1911. Zur Einweihung der Institute für Hüttenkunde an der Kgl. Technischen Hochschule zu Breslau. Stahl und Eisen, 31, 1565-1579.

Dürre, Ernst Friedrich, 1870. Wissenschaftlich-Technisches Handbuch des gesamten Gießereibetriebes. Leipzig: Felix.

Dürre, Ernst Friedrich, 1877. Das Probirlaboratorium des Polytechnicums zu Aachen. Zeitschrift des VDI, 21, 225-230.

Dürre, Ernst Friedrich, 1878. Bemerkungen zu einer Beurtheilung der deutschen technischen Erziehung seitens der Industrie. Annalen für Gewerbe und Bauwesen, 2, 73-82.

Dürre, Ernst Friedrich, 1882-1892. Die Anlage und der Betrieb der Eisenhütten. Ausführliche Zusammenstellung neuerer und bewährter Constructionen aus dem Bereiche der gesamten Eisen- und Stahl-Fabrikation unter Berücksichtigung aller Betriebs-Verhältnisse. 3 Bde. Leipzig: Baumgärtner.

Düwell, Kurt, 1970. Gründung und Entwicklung der Rheinisch-Westfälischen Technischen Hochschule Aachen bis zu ihrem Neuaufbau nach dem Zweiten Weltkrieg - Darstellung und Dokumente. In: Hans Martin Klinkenberg, Hg., Rheinisch-Westfälische Technische Hochschule Aachen 1870-1970. Stuttgart: Oscar Bek, 19-175.

Felt, Ulrike/Nowotny, Helga/Taschwer, Klaus, 1995. Wissenschaftsforschung. Eine Einführung. Frankfurt a. M.: Campus.

Felt, Ulrike, 2002. Wissenschaft, Politik und Öffentlichkeit - Wechselwirkungen und Grenzverschiebungen. In: Mitchell G. Ash und Christian H. Stifter, Hg., Wissenschaft, Politik und Öffentlichkeit. Von der Wiener Moderne zur Gegenwart. Wien: WUV-Universitätsverlag, 47-72.

Flachowsky, Sören, 2007. „Alle Arbeit des Instituts dient mit leidenschaftlicher Hingabe der deutschen Rüstung." Das Kaiser-Wilhelm-Institut für Eisenforschung als interinstitutionelle Schnittstelle kriegsrelevanter Wissensproduktion 1917-1945. In: Helmut Maier, Hg., Gemeinschaftsförderung, Bevollmächtigte und der Wissenstransfer. Die Rolle der Kaiser-Wilhelm-Gesellschaft im System kriegsrelevanter Forschung des Nationalsozialismus. Göttingen: Wallstein, 153-214.

Fleck, Ludwik, 1980. Entstehung und Entwicklung einer wissenschaftlichen Tatsache. Einführung in die Lehre vom Denkstil und Denkkollektiv. Frankfurt a. M.: Suhrkamp.

Goerens, Paul/Körber, Friedrich/Petersen, Otto, 1938. Nachruf Fritz Wüst. Stahl und Eisen, 58, 449-450.

Grübler, Arnulf, 1996. Time for a Change. On the Patterns of Diffusion of Innovation. Daedalus. Journal of the American Academy of Arts and Sciences, 125, 3, 19-42.

Guntau, Martin/Laitko, Hubert, Hg., 1987a. Der Ursprung der modernen Wissenschaften. Studien zur Entstehung wissenschaftlicher Disziplinen. Berlin: Akademie-Verlag.

Guntau, Martin/Laitko, Hubert, 1987b. Entstehung und Wesen wissenschaftlicher Disziplinen. In: Dies., Hg., Der Ursprung der modernen Wissenschaften. Studien zur Entstehung wissenschaftlicher Disziplinen. Berlin: AkademieVerlag, 17-89.

Hänseroth, Thomas/Mauersberger, Klaus, 1996. Das Dresdener Konzept zur Genese technikwissenschaftlicher Disziplinen - eine Bilanz. Dresdener Beiträge zur Geschichte der Technikwissenschaften, 24, 20-45.

Hänseroth, Thomas/Mauersberger, Klaus, 1998. Technikwissenschaften zwischen theoretischer Erkenntnis und Ingenieurtätigkeit. NTM, 6, 217-237.

Heymann, Matthias, 2005. „Kunst” und Wissenschaft in der Technik des 20. Jahrhunderts. Zur Geschichte der Konstruktionswissenschaft. Zürich: Chronos. 
Hoffmann, Walter, 1959. Bergakademie Freiberg. Frankfurt a. M.: Weidlich.

König, Wolfgang, 1995. Technikwissenschaften. Die Entstehung der Elektrotechnik aus Industrie und Wissenschaft zwischen 1880 und 1914. Chur: G+B Fakultas.

König, Wolfgang, 1999. Künstler und Strichezieher. Konstruktions- und Technikkulturen im deutschen, britischen, amerikanischen und französischen Maschinenbau zwischen 1850 und 1930. Frankfurt a. M.: Suhrkamp.

König, Wolfgang, 2006. Struktur der Technikwissenschaften. In: Gerhard Banse, Armin Grunwald, Wolfgang König und Günter Ropohl, Hg., Erkennen und Gestalten. Eine Theorie der Technikwissenschaften. Berlin: Edition Sigma, 3744.

Krebs, Stefan, 2007. „Leben heißt ein Kämpfer sein“. Zum Verhältnis von Wissenschaft und Öffentlichkeit am Beispiel der modernen Eisenhüttenkunde an der Technischen Hochschule Aachen. Berichte zur Wissenschaftsgeschichte, 30, 215-229.

Krebs, Stefan, 2009. Technikwissenschaft als soziale Praxis. Über Macht und Autonomie der Aachener Eisenhüttenkunde 1870-1914. Stuttgart: Franz Steiner.

Ledebur, Adolf, 1893. Rezension E. F. Dürre. Die neueren Koksöfen. Stahl und Eisen, 13, 89.

Maier, Helmut, 2007. Rüstungsforschung in der Kaiser-Wilhelm-Gesellschaft und das Kaiser-Wilhelm-Institut für Metallforschung 1900-1945/48. 2 Bde. Göttingen: Wallstein.

Manegold, Karl-Heinz, 1990. Alois Riedler. In: Wilhelm Treue und Wolfgang König, Hg., Berlinische Lebensbilder. Techniker. Berlin: Colloquium, 293-307.

Milkereit, Gertrud, 1977. Zum Verhältnis zwischen Forschung und Praxis im westdeutschen Eisenhüttenwesen zwischen 1870 und 1910 am Beispiel des Lehrstuhls für Allgemeine Hüttenkunde an der Technischen Hochschule Aachen und seine Beziehungen zum Verein Deutscher Eisenhüttenleute. Technikgeschichte, 44, 293-301.

Redtenbacher, Ferdinand, 1852. Prinzipien der Mechanik und des Maschinenbaus. Mannheim: Bassermann.

Reuleaux, Franz, 1875. Theoretische Kinematik. Grundzüge einer Theorie des Maschinenwesens. Braunschweig: Vieweg.

Richter, Siegfried H., 1984. Methodenentwicklung und Methodenstreit in Lehre und Forschung der mechanischen Technologie im 19. Jahrhundert in Deutschland. Dresdener Beiträge zur Geschichte der Technikwissenschaften, 10, 3-42.

Röntgen, Paul, 1955. Wilhelm Borchers. In: Historische Kommission bei der Bayerischen Akademie der Wissenschaften, Hg., Neue Deutsche Biographie, Bd. 2. Berlin: Duncker \& Humblot, 457-458.

Schenck, Hermann, 1971. Probleme und Brennpunkte der Forschung in Nordrhein-Westfalen. In: Karl Arnold, Hermann Schenck und Leo Brandt, Hg., Wege und Ziele der Forschung in Nordrhein-Westfalen. Opladen: Westdeutscher Verlag, 13-27.

Schlink, Joseph, 1878a. Die Bestrebungen der technischen Lehrinstitute und die Anforderungen des praktischen Lebens. Annalen für Gewerbe und Bauwesen, 2, 8-12/33-38.

Schlink, Joseph, 1878b. Die Bestrebungen der technischen Lehrinstitute und die Anforderungen des praktischen Lebens II. Annalen für Gewerbe und Bauwesen, 2, 187-191/221-228.

Schwarz, Karl, Hg., 2000. 1799-1999. Von der Bauakademie zur Technischen Universität Berlin. Geschichte und Zukunft. Berlin: Ernst.

TH Aachen, Hg., 1879. Die Chemischen Laboratorien der königlichen rheinischwestfälischen Technischen Hochschule zu Aachen. Aachen: Mayer.

Vorstand des Vereins Deutscher Eisenhüttenleute, 1903. Das höhere eisenhüttenmännische Unterrichtswesen in Preussen. Stahl und Eisen, 23, 857859. 
Wedding, Hermann, 1878. Uebungen im Entwerfen von Eisenhütten-Anlagen an den technischen Hochschulen. Annalen für Gewerbe und Bauwesen, 2, 270272.

Weingart, Peter, Hg., 1974. Wissenschaftssoziologie II. Determinanten wissenschaftlicher Entwicklung. Frankfurt a. M.: Athenäum.

Wengenroth, Ulrich, 1993. Eisen, Stahl und Buntmetalle. In: Wilhelm Dettmering und Armin Hermann, Hg., Technik und Wirtschaft. Düsseldorf: VDI, 197-208.

Wüst, Fritz, 1905. Nachruf Ernst Friedrich Dürre. Stahl und Eisen, 25, 383-384.

Wüst, Fritz, Hg., 1908-1913. Mitteilungen aus dem Eisenhüttenmännischen Institut der Königlichen Technischen Hochschule Aachen. Bd. 2-5. Halle a. S.: Knapp.

Wüst, Fritz, 1909. Über die Entwicklung des Zustandsdiagramms der EisenKohlenstofflegierungen. Zeitschrift für Elektrochemie, 15, 565-584.

\section{Stefan Krebs}

Department of Technology,

Innovation and Society

Eindhoven University of Technology

IPO 1.16, P.O. Box 513

5600 MB Eindhoven

Niederlande

Email: s.krebs@tue.nl 